\title{
On the factorization of certain probability distributions
}

\author{
By Harald Cramér
}

1. The object of this Note is to show that a large class of probability distributions of the type known as infinitely divisible have divisors which are not infinitely divisible. Among these distributions are, in particular, the Pearson Type III distribution and all non-normal stable distributions.

In the first two paragraphs, we briefly recall some known definitions and results. The main theorem will then be stated and proved in paragraph 3 .

If $\xi$ is a random variable, the probability of the relation $\xi \leqq x$ is a function $F(x)$ of the real variable $x$. The function $F(x)$. which determines the probability distribution of the variable $\xi$, is known as the distribution function (d.f.) of $\xi$. In order that a given function $F(x)$ should be the d.f. of some random variable, it is necessary and sufficient that $F(x)$ should be never decreasing and everywhere continuous to the right, and such that $F(-\infty)=0$ and $F(+\infty)=1$. The function

$$
\varphi(t)=\int_{-\infty}^{\infty} e^{i t x} d F(x)
$$

is called the characteristic function (c.f.) of the distribution. To every d.f. $F(x)$, there is one and only one c.f. $\varphi(t)$, uniquely defined for all real $t$.

Suppose now that the variables $\xi_{1}$ and $\xi_{2}$ are independent in the ordinary probability sense, and consider the sum $\xi=\xi_{1}+\xi_{2}$. Let the d.f:s of the variables $\xi, \xi_{1}$ and $\xi_{2}$ be $F, F_{1}$ and $F_{2}$, while the corresponding c.f:s are $\varphi, \varphi_{1}$ and $\varphi_{2}$. We then have

$$
F(x)=\int_{-\infty}^{\infty} F_{3}(x-t) d F_{2}(t)=\int_{-\infty}^{\infty} F_{2}(x-t) d F_{1}(t) .
$$

We shall say that $F$ is composed of $F_{1}$ and $F_{2}$, and use the abbreviated notation

$$
F=F_{1} * F_{2}=F_{2} * F_{1} \text {. }
$$

To this symbolic multiplication of the d.f:s corresponds a real multiplication of the c.f:s. We have, in fact, $\varphi(t)=\varphi_{1}(t) \varphi_{2}(t)$. The operation of composition is commutative and associative, so that any symbolic product $F=F_{1} * \cdots * F_{n}$ is uniquely defined and independent of the order of the factors. The symbolic product of $n$ identical factors will be written as a symbolic $n$th power: $F^{[n]}$. 
Thus the class of all $\mathrm{d}$. $\mathrm{f}: \mathrm{s}$ forms a semigroup with respect to the operation of composition. The unit element of this semigroup is the d.f.

$$
E(x)= \begin{cases}0 & \text { for } \quad x<0 \\ 1 & \text { for } \quad x \geqq 0\end{cases}
$$

which is the d.f. of a "variable» $\xi$ which always takes the value 0 . The on? divisors of the unit are the d.f:s of the form $E(x-m)$, where $m$ is a constant. We have, in fact $E(x)=E(x-m) * E(x+m)$. The corresponding relation between the c.f:s is $1=e^{m i t} \cdot e^{-m i t}$.

A factorization of the probability distribution defined by $F(x)$ is any representation $F=F_{1} * \cdots * F_{n}$, where $n>1$, and no $F_{r}$ is a divisor of the unit. Each $F_{r}$ is then said to be a divisor of $F$. When $F$ has no factorization, it is called indecomposable. A simple case of an indecomposable d. f. is

$$
F(x)=p E(x)+q E(x-1)
$$

where $p$ and $q$ are positive constants such that $p+q=1$.

2. The probability distribution defined by $F(x)$ is called infinitely divisible, if to every $n=1,2, \ldots$ we can find a d.f. $G$ such that $F=G^{[n]}$. The properties of infinitely divisible distributions have been studied by several authors, notably by Paul LÉvy, who has shown (cf e. g. 3) that the logarithm of the c.f. $\varphi(t)$ of any infinitely divisible distribution can be written in the form

$$
\log \varphi(t)=m i t-\frac{1}{2} \sigma^{2} t^{2}+\int_{-\infty}^{\infty}\left(e^{i t x}-1-\frac{i t x}{1+x^{2}}\right) d M(x),
$$

where $m$ and $\sigma^{2} \geqq 0$ are real constants, while $M(x)$ is real and never decreasing in each of the intervals $(-\infty, 0)$ and $(0,+\infty)$, and such that $M(-\infty)=M(+\infty)=0, \int_{-1}^{1} x^{2} d M(x)<\infty$. Further, any choice of $m, \sigma$ and $M(x)$ consistent with these conditions yields an infinitely divisible distribution, and the representation of $\log \varphi(t)$ in the form (1) is unique.

The class of infinitely divisible distributions includes many important types of distributions occurring in various applications. Thus for $M(x)=0$ we have the normal distribution, and for $\sigma=0, M(x)=\lambda E(x-1)-\lambda E(x)$ the Poisson distribution. For $\sigma=0, M(x)=0$ for $x<0$ and $M^{\prime}(x)=\lambda x^{-1} e^{-\alpha x}$ for $x>0$, we obtain the Pearson Type III distribution, and finally for $\sigma=0$, $M^{\prime}(x)=A|x|^{-\alpha-1}$ for $x<0$ and $M^{\prime}(x)=B x^{-c-1}$ for $x>0$, where $0<\alpha<2$, we obtain the non-normal stable distributions.

It follows from the above that the problem of finding all possible factorizations of an infinitely divisible d.f. $F$ can be completely solved, as long as we restrict ourselves to factors which are themselves infinitely divisible. In fact, if $F=F_{1} * \cdots * F_{n}$, where every $F_{r}$ is infinitely divisible, the logarithm of the c. f. $\varphi_{r}$ corresponding to $F_{r}$ can be written in the form (1), and we must have $m=\sum m_{r}, \sigma^{2}=\sum \sigma_{r}^{\prime}, M(x)=\sum M_{r}(x)$. Conversely, any choice of the $m_{r}$, 
$\sigma_{r}$ and $M_{r}(x)$ consistent with these conditions yields a factorization of $F$, such that all the factors are infinitely divisible.

In the two particular cases of the normal and the Poisson distributions, it is known (CramÉr, 1, p. 52, and RaIKov, 5) that the factorizations obtained in this way remain the only possible ones, even if we remove the restriction that the factors $F_{r}$ should be infinitely divisible. Thus any divisor of a normal distribution is itself normal, and similarly for the Poisson distribution.

On the other hand, it has been shown by examples (cf e.g. LÉvY, 3 and 4) that there exist infinitely divisible d.f:s $F$ such that $F$ can be factorized into $F=F_{1} * F_{2}$, where at least one of the factors is not infinitely divisible. This case occurs e. g. when $F$ is the symbolic product of a certain number of appropriately chosen Poisson distributions.

The object of this Note is to show that, in fact, this property holds for a large class of infinitely divisible distributions.

3. Consider, an infinitely divisible d.f. $F$, and let the logarithm of the corresponding c.f. $\varphi$ be represented in the form (1). The function $M(x)$, being never decreasing, has almost everywhere a finite derivative $M^{\prime}(x) \geqq 0$. We now proceed to prove the following theorem:

Suppose that we can find two positive constants $k$ and $c$ such that $M^{\prime}(x)>k$ almost everywhere in at least one of the two intervals $(-c, 0)$ and $(0, c)$. Then $F$ has a divisor which is not infinitely divisible.

Thus in particular the Type III distribution, as well as any non-normal stable distribution, has a divisor which is not infinitely divisible.

In order to prove the theorem, we may restrict ourselves to the case when the condition is satisfied in the interval $(0, c)$. It then follows from the preceding paragraph that $F$ has an infinitely divisible divisor $G$ defined by taking in the expression (1) of the c.f. $m=\sigma=0, M^{\prime}(x)=k$ for $0<x<c$, and $M^{\prime}(x)=0$ elsewhere. Thus we have only to show that $G$ has a non-infinitely divisible divisor.

Denoting by $\gamma(t)$ the c.f. corresponding to $G$, we have according to (1)

$$
\log \gamma(t)=k \int_{0}^{c}\left(e^{i t x}-1-\frac{i t x}{1+x^{2}}\right) d x=\log \gamma_{1}(t)+\log \gamma_{2}(t,),
$$

where we take

$$
\begin{gathered}
\log \gamma_{r}(t)=k \int_{0}^{c}\left(e^{i t x}-1-\frac{i t x}{1+x^{2}}\right) \alpha_{r}(x) d x, \quad(r=1,2), \\
\alpha_{1}(x)=\left\{\begin{array}{cl}
-\varepsilon & \text { for }\left(\frac{1}{2}-\varepsilon\right) c<x<\left(\frac{1}{2}+\varepsilon\right) c, \\
1 & \text { elsewhere in }(0, c) . \\
0 & \text { outside }(0, c),
\end{array}\right. \\
\alpha_{2}(x)=\left\{\begin{array}{cc}
1+\varepsilon & \text { for }\left(\frac{1}{2}-\varepsilon\right) c<x<\left(\frac{1}{2}+\varepsilon\right) c, \\
0 & \text { elsewhere. }
\end{array}\right.
\end{gathered}
$$

Here $\varepsilon$ denotes a constant such that $0<\varepsilon<\frac{1}{2}$. 
HARALD CRAMÉR, On the factorization of certain probability distributions

It follows from (1) that $\gamma_{2}(t)$ is the c. f. of an infinitely divisible d. f. $G_{2}$. We shall now show that, if $\varepsilon$ is sufficiently small, $\gamma_{1}(t)$ will be the c. f. of a non-infinitely divisible d.f. $G_{1}$. Then by (2) we have $G=G_{1} * G_{2}$, and our theorem will be proved.

For every real $x$, we define the functions $\beta_{1}(x), \beta_{2}(x), \ldots$ by writing

$$
\begin{aligned}
& \beta_{1}(x)=z_{2} \alpha_{1}(x), \\
& \beta_{2}(x)=\int_{0}^{x} \beta_{1}(x-t) \beta_{1}(t) d t, \\
& \beta_{n}(x)=\int_{0}^{x} \beta_{n-1}(x-t) \beta_{1}(t) d t .
\end{aligned}
$$

We then have $\beta_{n}(x)=0$ everywhere outside $(0, n c)$, and it is well known that we have

$$
\int_{0}^{n} e^{i t x} \beta_{n}(x) d x=k^{n}\left(\int_{0}^{e} e^{i t x} \alpha_{1}(x) d x\right)^{n} .
$$

Further, $\left|\beta_{n}(x)\right| \leqq k^{n} c^{n-1}$ for all $x$, and it follows that

$$
\int_{0}^{\infty} e^{i t x} \sum_{1}^{\infty} \frac{\beta_{n}(x)}{n !} d x=\sum_{1}^{\infty} \frac{1}{n !} \int_{0}^{n c} e^{i t x} \beta_{n}(x) d x=\exp \left(k \int_{0}^{e} e^{i t x} \alpha_{1}(x) d x\right)-1
$$

Writing

$$
x=k \int_{0}^{c} \alpha_{1}(x) d x, \quad \lambda=k \int_{0}^{c} \frac{x}{1+x^{2}} \alpha_{1}(x) d x
$$

we now obtain from (3) and (5)

where

$$
\gamma_{1}(t)=e^{-x \cdots, i t}\left(1+\int_{0}^{\infty} e^{i t x} \sum_{1}^{\infty} \frac{\beta_{n}(x)}{n !} d x\right)=\int_{-\infty}^{\infty} e^{i t x} d G_{1}(x)
$$

$$
G_{1}(x)=e^{-x}\left[E(x+\lambda)+\int_{-\infty}^{x} \sum_{1}^{\infty} \frac{\beta_{n}(u+\lambda)}{n !} d u\right]
$$

Obviously $G_{1}(-\infty)=0$, while from (3) we obtain $G_{1}(+\infty)=\gamma_{1}(0)=1$. In order to prove that $G_{1}(x)$ is a d.f., we now only have to show that it is never decreasing, i. c. that $\sum_{1}^{\infty} \beta_{n}(x) / n ! \geqq 0$ for all $x$. 
Consider first $\beta_{1}(x)$, which is everywhere non-negative, except between the limits $\left(\frac{1}{2} \pm \varepsilon\right) c$, where it takes the value $-k \varepsilon$. Further, as $\varepsilon \rightarrow 0$, it is obvious that $\beta_{2}(x)$ tends to $k^{2}(c-|c-x|)$ uniformly in $0<x<2 c$. Also it is easily seen that for all sufficiently small $\varepsilon$ we have $\beta_{2}(x) \geqq 0$ and $\beta_{3}(x) \geqq 0$ for all $x$. It follows that we can find $\varepsilon_{0}$ such that for $\varepsilon=\varepsilon_{0}$ and for all $x$

$$
\begin{aligned}
& \beta_{1}(x)+\frac{1}{2 !} \beta_{2}(x)+\frac{1}{3 !} \beta_{3}(x) \geqq 0, \\
& \beta_{4}(x)=\int_{0}^{x} \beta_{2}(x-t) \beta_{2}(t) d t \geqq 0, \\
& \beta_{5}(x)=\int_{0}^{x} \beta_{3}(x-t) \beta_{2}(t) d t \geqq 0,
\end{aligned}
$$

and hence $\sum_{1}^{\infty} \frac{\beta_{n}(x)}{n !} \geqq 0$. Thus $G_{1}$ is a d.f.

It now only remains to show that $G_{1}$ is not infinitely divisible. In fact, if $G_{1}$ were infinitely divisible. $\log \gamma_{1}(t)$ could be expressed in the form (1) with a never decreasing $M(x)$. Now since the derivative $M^{\prime}(x)$ exists almost everywhere and is $\geqq 0$, and since the representation (1) is unique, it follows from (2) and (3) that we should have almost everywhere in $(0, c)$

$$
k=M^{\prime}(x)+k \alpha_{2}(x) \geqq k \alpha_{2}(x),
$$

but this is obviously inconsistent with (4). Thus $G_{1}$ cannot be infinitely divisible, and our theorem is proved.

4. By a theorem due to KHIntchnse (2), any d. f. which is not infinitely divisible has an indecomposable divisor. Writing the function $G$ of the preceding paragraph in the form $G=G^{\left[\frac{1}{2}\right]} * G^{\left[\frac{1}{4}\right]} * \cdots$, and applying first the argument of the preceding paragraph and then KHINTCHinE's theorem to each factor, we obtain the following result:

Any infinitely divisible d.t. F satisfying the conditions of the preceding theorem is divisible by the product of an infinite sequence of indecomposable d. $f: s$.

REFERENCES : 1. Cramér, H., Random variables and probability distributions. Cambridge Tracts in Mathematics, Cambridge 1937. - 2. Khintchine, A., Contribution $\dot{x}$ l'arithmétique des lois de distribution. Bull. Math. Univ. Moscou. 1 (1937), p. 6. 3. Lévy, P., Théorie de l'addition des variables aléatoires. Paris 1937. - 4. _-, L'arithmétique des lois de probabilités. Journ. Math. Pures et Appl, 17 (1938), p. 17. - 5. Raikov, D., On the composition of Poisson laws. C. R. Acad. Scient. URSS, 14 (1937), p. 9. 